\title{
RELIABILITY REQUIRED FOR HERITAGE STRUCTURES
}

\author{
MILAN HOLICKÝ \\ Klokner Institute, Czech Technical University in Prague, Czech Republic.
}

\begin{abstract}
The required reliability levels recommended in national and international documents for new and existing structures vary within a broad range, while the reference to relevant costs and failure consequences is mentioned only very vaguely. This contribution attempts to clarify the relationship between the reliability levels required for existing structures, construction costs, failure consequences, reference period, the design working life and the discount rate. The theoretical study is based on probabilistic optimization of the total costs taking into account construction value including historical and artistic value, obsolescence of existing structures, discount rate and time to failure. However, historical and artistic values of some heritage structures are often difficult to assess in monetary units and then the expert judgements are to be utilized. It appears that the optimal reliability level depends primarily on the construction costs, failure costs, and relative cost for improving structural safety, and less significantly on the discount rate and the time to failure.

Keywords: artistic values, discount rate, heritage structures, optimization, reliability, total costs.
\end{abstract}

\section{INTRODUCTION}

The target reliability levels recommended in various national and international documents for new structures are inconsistent in terms of the values and the criteria according to which the appropriate values are to be selected. Almost no recommendations are available for temporary structures. In general, optimum reliability levels can be obtained by considering both the cost of the structure and the expected cost of failure over the design working life.

The design working life is understood as an assumed period of time for which a structure is to be used for its intended purpose without any major repair work being necessary. Indicative values of design working life (10 to 100 years for different types of new structures) are given in EN 1990 (2002) [1]. Recommended values of reliability indexes are given for two reference periods, 1 year and 50 years (Table 1), without any explicit link to the design working life that generally differs from the reference period, while no specific indicative values are available for temporary structures.

It should be emphasized that the reference period is understood as a chosen period of time used as a basis for statistically assessing the time variant basic random variables, and the corresponding probability of failure. The concept of reference period is therefore fundamentally different from the concept of design working life. Confusion is often caused when the difference between these two concepts is not recognized.

It should be recognized that the couple of $\beta$ values (for 1 year and 50 years) given in Table 1 for each reliability class correspond to the same reliability level. Practical application of these values, however, depends on the time period considered in the verification, which may be linked to available probabilistic information concerning time variant basic variables (imposed load, wind, earthquake, etc.). It should be noted that the reference period of 50 years is also accepted as the design working life for common structures (see the discussion by Diamantidis (2009) [2]).

For example, considering a structure of reliability class 2 having a design working life of 50 years, the reliability index $\beta=3.8$ should be used, provided that probabilistic models of basic variables are available for this period. The same reliability level is achieved when a 
Table 1: Reliability classification in accordance with EN 1990 (2002) [1].

\begin{tabular}{|c|c|c|c|c|}
\hline \multirow{2}{*}{$\begin{array}{l}\text { Reliability } \\
\text { classes }\end{array}$} & \multirow{2}{*}{$\begin{array}{l}\text { Consequences of } \\
\text { structural failure }\end{array}$} & \multicolumn{2}{|c|}{$\begin{array}{l}\text { Reliability index } \beta \\
\text { for reference period }\end{array}$} & \multirow[t]{2}{*}{$\begin{array}{l}\text { Examples of buildings and } \\
\text { civil engineering works }\end{array}$} \\
\hline & & 1 year & 50 years & \\
\hline RC3 - high & High & 5.2 & 4.3 & Bridges, public buildings \\
\hline RC2 - normal & Medium & 4.7 & 3.8 & Residences and offices \\
\hline $\mathrm{RC} 1-$ low & Low & 4.2 & 3.3 & Agricultural buildings \\
\hline
\end{tabular}

reference period of 1 year, and a target of $\beta=4.7$ are applied using the theoretical models for a reference period of 1 year. Thus, when designing a structural member, similar dimensions (reinforcement area) would be obtained considering $\beta=4.7$ and basic variables related to 1 year or $\beta=3.8$ and basic variables related to 50 years.

A more detailed recommendation concerning the target reliability is provided by ISO 2394 (1998) [3], where the target reliability indexes are indicated for the whole design working life without any restriction concerning its length, and are related not only to the consequences, but also to the relative costs of safety measures (Table 2 ).

Similar recommendations are provided in the JCSS (2001) [4] Probabilistic Model Code (Table 3) based on the previous study of Rackwitz (2000) [5]. The recommended target reliability indexes are also related to both the consequences and to the relative costs of safety measures, though for a reference period of 1 year. The consequence classes in JCSS (2001) [4] (similar to EN 1990, 2002 [1]) are linked to the ratio $\rho$ defined as the ratio $\left(C_{\mathrm{str}}+C_{\mathrm{f}}\right) / C_{\mathrm{str}}$ of the total cost induced by a failure (cost of construction $C_{\text {str }}$ plus direct failure costs $C_{\mathrm{f}}$ ) to the construction $\operatorname{cost} C_{\text {str }}$ as follows:

Table 2: Examples of life-time target reliability indexes $\beta$ in accordance with ISO 2394 (1998) [3].

\begin{tabular}{lcccc}
\hline \multirow{2}{*}{$\begin{array}{l}\text { Relative costs of safety } \\
\text { measures }\end{array}$} & \multicolumn{4}{l}{ Consequences of failure } \\
\cline { 2 - 5 } & small & Some & moderate & great \\
\hline High & 0 & 1.5 & 2.3 & 3.1 \\
Moderate & 1.3 & 2.3 & 3.1 & 3.8 \\
Low & 2.3 & 3.1 & 3.8 & 4.3 \\
\hline
\end{tabular}

Table 3: Tentative target reliability indexes $\beta$ (and associated target failure rates) related to 1 -year reference period and ultimate limit states in accordance with JCSS (2001) [4].

\begin{tabular}{llll}
\hline $\begin{array}{l}\text { Relative costs of } \\
\text { safety measures }\end{array}$ & $\begin{array}{l}\text { Minor consequences } \\
\text { of failure }\end{array}$ & $\begin{array}{l}\text { Moderate consequences } \\
\text { of failure }\end{array}$ & $\begin{array}{l}\text { Large consequences } \\
\text { of failure }\end{array}$ \\
\hline $\begin{array}{l}\text { Large } \\
\text { Normal }\end{array}$ & $\beta=3.1\left(p \approx 10^{-3}\right)$ & $\beta=3.3\left(p \approx 5 \times 10^{-4}\right)$ & $\beta=3.7\left(p \approx 10^{-4}\right)$ \\
Small & $\beta=3.7\left(p \approx 10^{-4}\right)$ & $\beta=4.2\left(p \approx 10^{-5}\right)$ & $\beta=4.4\left(p \approx 5 \times 10^{-6}\right)$ \\
\hline
\end{tabular}


- Class 1 Minor Consequences: $\rho$ is less than approximately 2; risk to life, given a failure, is small to negligible and the economic consequences are small or negligible (e.g. agricultural structures, silos, masts);

- Class 2 Moderate Consequences: $\rho$ is between 2 and 5; risk to life, given a failure, is medium and the economic consequences are considerable (e.g. office buildings, industrial buildings, apartment buildings);

- Class 3 Large Consequences: $\rho$ is between 5 and 10; risk to life, given a failure, is high, and the economic consequences are significant (e.g. main bridges, theatres, hospitals, high-rise buildings).

However, it is not quite clear what is meant in JCSS (2001) [4] by 'the direct failure costs'. This term indicates that there may be some other 'indirect costs' that may affect the total expected cost. Here it is assumed that the failure costs $C_{\mathrm{f}}$ cover all additional direct and indirect costs (except the structural costs $C_{\text {str }}$ ) induced by the failure. The structural costs are considered separately and related to the costs needed for an improvement of safety (costs per unit of decision parameter $C_{1}$ ).

Both the documents ISO 2394 (1998) [3] and JCSS (2001) [4] seem to recommend reliability indexes that are lower than those given in EN 1990 (2002) [1] even for the 'small relative costs' of safety measures. It should be noted that EN 1990 (2002) [1] gives the reliability indexes for two reference periods ( 1 and 50 years) that may be accepted as the design working life for common structures (see also the discussion provided by Diamantidis (2009) [2]). ISO 2394 (1998) [3] recommends indexes for 'life-time, examples', thus related to the design working life, without any restrictions, while Probabilistic Model Code by JCSS (2001) [4] provides reliability indexes for the reference period of 1 year.

However, a clear link between the design working life and the target reliability level is not apparent from any of the above-mentioned documents. Thus, it is not clear which target reliability index should be used for a given design working life different from 50 years (say 10 years).

A new promising approach to specify the target reliability based on the concept of Life Quality Index (Fischer et al., 2012) [6] is considered in an on-going revision of the International Standard ISO 2394 (1998) [3].

The basic aim of this contribution is to clarify the link between the design working life and the reliability index, and to provide guidance for specification of the target reliability level for a given design working life. The submitted theoretical study based on probabilistic optimization is supplemented by practical recommendations. This contribution is an extension of the previous study by Holicky and Retief (2011) and Holicky [7, 8].

\section{GENERAL PRINCIPLES OF PROBABILISTIC OPTIMIZATION}

Probabilistic optimization is based on a fundamental form of the objective function (not covering monitoring and maintenance) expressed as the present value of the total expected cost $C_{\text {tot }}(x, o, q, n)$

$$
C_{\mathrm{tot}}(x, o, q, n)=C_{\mathrm{str}} \sum_{1}^{n} P_{\mathrm{f}}(x, i) Q(o, i)+C_{\mathrm{f}} \sum_{1}^{n} P_{\mathrm{f}}(x, i) Q(q, i)+C_{0}+x C_{1}
$$

The cost of construction $C_{\text {str }}$ including artistic value is discounted as it is paid in the future after number of years $i$. Here, $x$ denotes the decision parameter of the optimization (a parameter 
of structural resistance), $o$ is the annual obsolescence (oldness) rate of heritage structure enhanced by annual discount rate $q$.

The cost of failure $C_{\mathrm{f}}$ including relevant artistic values is also discounted as it is paid after number of years $i, q$ is the annual discount rate (without obsolescence rate $o$ ), e.g. 0.03, an average long run value of the real annual discount rate in European countries, $n$ is the number of years to the failure, which may differ from the design working life (specified usually as 50 or 100 years).

Further, $P_{\mathrm{f}}(x, i)$ is the failure probability in year $i, Q(o, i)$ is the discount factor dependent on the annual obsolescence rate $o, Q(q, i)$ is the discount factor dependent on the annual discount rate $q$ and the number of years $i, C_{0}$ is the initial cost of intervention independent of the decision parameter $x$ and failure (a quantity not affecting the optimization) and $C_{1}$ is the cost per unit of the decision parameter $x$ (a structural parameter quantity affecting the structural resistance and optimization).

Note that the design working life may generally differ from the time to failure denoted by the number of years $n$ and considered here as an independent variable affecting the probability of failure. Maintenance and possible repair of the structure is not included in the objective function (1), and these aspects are to be considered in further studies. Assuming independent failure events in subsequent years, the annual probability of failure $P_{\mathrm{f}}(x, i)$ in year $i$ may be approximated by the geometric sequence

$$
P_{\mathrm{f}}(x, i)=p(x)(1-p(x))^{i-1}
$$

The initial annual probability of failure $p(x)$ is dependent on the decision parameter $x$. Note that annual failure probabilities can be assumed to be independent when failure probabilities are chiefly influenced by time-variant loads (climatic actions, traffic loads, accidental loads). Then the failure probability $P_{\mathrm{f} n}(x)$ during $n$ years can be estimated by the sum of the sequence $P_{\mathrm{f}}(x, i)$, that can be expressed as

$$
P_{\mathrm{f} n}(x, n)=1-(1-p(x))^{n} \approx n p(x)
$$

Note that the approximation indicated in eqn (3) is fully acceptable for small annual probabilities $p(x)<10^{-3}$.

The discount factor of the present value of the expected future costs in year $i$ is considered in the usual form as

$$
Q(q, i)=1 /(1+q)^{i}
$$

Thus, the cost of malfunctioning $C_{\mathrm{f}}$ is discounted by the factor $Q(q, i)$ depending on the discount rate $q$ and the point in time (year number defined as $i$ ) when the loss of structural utility occurs.

Considering eqns (2) and (4) the total costs $C_{\text {tot }}(x, q, n)$ described by eqn (1) may be written in a simplified form as

$$
C_{\text {tot }}(x, o, q, n)=C_{\text {str }} P Q(x, o, n)+C_{\mathrm{f}} p(x) P Q(x, q, n)+C_{0}+x C_{1}
$$

Here, the total sum of expected structural cost after $n$ years depends on the present structural $\operatorname{cost} C_{\text {str }}$, the annual probability $p(x)$ and on the sum of the geometric sequence having the quotient $(1-p(x)) /(1+o)$, denoted as the time factor $P Q(x, o, n)$. Similarly, malfunctioning costs after $n$ years is dependent on the product of the present value of malfunction $\cos t C_{\mathrm{f}}$, the annual probability $p(x)$ and a sum of the geometric sequence having the quotient $(1-p(x)) /$ $(1+q)$, denoted as the time factor $P Q(x, q, n)$ : 


$$
P Q(x, o, n)=\frac{1-\left[\frac{1-p(x)}{1+o}\right]^{n}}{1-\left[\frac{1-p(x)}{1+o}\right]}, \quad P Q(x, q, n)=\frac{1-\left[\frac{1-p(x)}{1+q}\right]^{n}}{1-\left[\frac{1-p(x)}{1+q}\right]}
$$

In general, the total cost $C_{\text {tot }}(x, o, q, n)$ depends on the costs $C_{0}, C_{1}, C_{\text {str }}, C_{\mathrm{f}}$, the annual probability of failure $p(x)$, the oldness rate $o$, on the discount rate $q$ and the number of years $n$. Note that for small probabilities of failure $p(x)$ (for appropriate structural parameter $x$ ) and very small (zero) rates $o$ and $q$, the time factor $P Q(x, o, n) \approx P Q(x, q, n) \approx n$. Variation of the time factor $P Q(x, o, n)$ with $n$ for $o=0,0.03,0.06$ and 0.13 is shown in Fig. 1. The same variation holds for the time factor $P Q(x, q, n)$.

The necessary condition for the minimum of the total cost follows from (1) as

$$
\begin{aligned}
& \frac{\partial C_{\mathrm{tot}}(x, q, n)}{\partial x}=C_{\mathrm{str}} \sum_{i=1}^{n} Q(o, i)\left[\frac{\partial P_{\mathrm{f}}(x, i)}{\partial x}\right]_{x=x_{\mathrm{opt}}}+ \\
& C_{\mathrm{f}} \sum_{i=1}^{n} Q(q, i)\left[\frac{\partial P_{\mathrm{f}}(x, i)}{\partial x}\right]_{x=x_{\mathrm{opt}}}+C_{1}=0
\end{aligned}
$$

Equation (7) represents a general form of the necessary condition for the minimum of total $\operatorname{cost} C_{\text {tot }}(x, q, n)$, the optimum value $x_{\text {opt }}$ of the parameter $x$ and the optimum annual probability of failure $p_{\mathrm{opt}}=p\left(x_{\mathrm{opt}}\right)$. The optimum probability for the total design working life $T_{\mathrm{d}}=n$ years follows from eqn (7) as

$$
\begin{aligned}
& \frac{C_{\text {str }}}{C_{1}} \sum_{i=1}^{n} Q(q, i)\left[\frac{\partial P_{\mathrm{f}}(x, i)}{\partial x}\right]_{x=x_{\mathrm{opt}}}+ \\
& \frac{C_{\mathrm{f}}}{C_{1}} \sum_{i=1}^{n} Q(q, i)\left[\frac{\partial P_{\mathrm{f}}(x, i)}{\partial x}\right]_{x=x_{\mathrm{opt}}}=-1
\end{aligned}
$$

Equation (8) represents a simplified form of the necessary condition for the minimum of total cost $C_{\text {tot }}(x, o, q, n)$, the optimum value $x_{\text {opt }}$ of the parameter $x$ and the optimum annual

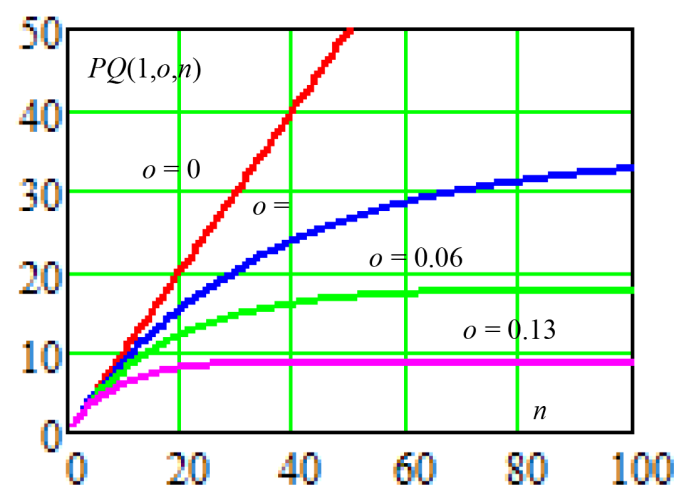

Figure 1: Variation of the time factor $P Q(x, o, n)$ with $n$ for $o=0,0.03,0.06$ and 0.13 . 
probability of failure $p_{\mathrm{opt}}=p\left(x_{\mathrm{opt}}\right)$. The optimum probability for the total design working life $T_{\mathrm{d}}=n$ years follows from eqn $(3)$ as

$$
P_{\mathrm{f} n, \mathrm{opt}}=1-\left(1-p_{\mathrm{opt}}\right)^{n} \approx n p_{\mathrm{opt}}
$$

The corresponding optimum reliability index $\beta_{\mathrm{opt}}=-\Phi^{-1}\left(P_{\mathrm{f} n, \mathrm{opt}}\right)$. These quantities are in general dependent on the cost ratios $C_{\mathrm{str}} / C_{1}$ and $C_{\mathrm{f}} / C_{1}$, rates $o$ and $q$, and on the number of years $n$.

3 FAILURE PROBABILITY OF A GENERIC STRUCTURAL MEMBER

Consider a generic structural member described by the limit state function $Z(x)$ as

$$
Z(x)=x f-(G+Q)
$$

Here, $x$ denotes a deterministic structural parameter (e.g. the cross-section area), $f$ the strength of the material, $G$ the load effect due to permanent load and $Q$ the load effect due to variable load. Theoretical models of the random quantities $f, G$ and $Q$ considered in the following example are given in Table 4 (adopted from JCSS (2001) [4] and Holicky (2009) [9]).

Considering the theoretical models given in Table 4, the reliability margin $Z(x)$ may be well approximated by the normal distribution $\Phi Z(x)$ that provides sufficient accuracy. The annual failure probability $p(x)$ is then given as

$$
p(x)=\Phi Z(x)(Z(x)=0)
$$

In eqn (11) the normal distribution is evaluated for $Z(x)=0$; then for $x=1$ and $n=50$ the probability $P_{\mathrm{f} n}(1,50) \approx 6.710^{-5}$ and corresponding reliability index $\beta \approx 3.8$.

\section{AN EXAMPLE}

The following example illustrates the general principles, as well as a special case of probabilistic optimization. To simplify the analysis, the total costs $C_{\text {tot }}(x, o, q, n)$ given by eqn (5) are transformed to the standardized form $\kappa_{\text {tot }}(x, o, q, n)$ given as

$$
\begin{aligned}
& \kappa_{\text {tot }}(x, o, q, n)=\frac{C_{\text {tot }}(x, o, q, n)-C_{0}}{C_{1}}= \\
& p(x)\left[\frac{C_{\text {str }}}{C_{1}} P Q(x, o, n)+\frac{C_{\mathrm{f}}}{C_{1}} P Q(x, q, n)\right]+x
\end{aligned}
$$

The annual probability of failure $p(x)$ considered here for a general structural member is given by eqn (11). However, the following procedure may be applied for any relevant dependence of the failure probability $p(x)$ expressed as a function of a suitable structural parameter $x$.

Table 4: Theoretical models of the random variables $f, G$ and $Q$ (annual extremes).

\begin{tabular}{lllll}
\hline Variables & Distribution & Mean & Standard deviation & Coefficient of variation \\
\hline$f$ & Lognormal & 100 & 10 & 0.10 \\
$G$ & Normal & 35 & 3,5 & 0.10 \\
$Q$ & Gumbel & 10 & 5 & 0.50 \\
\hline
\end{tabular}




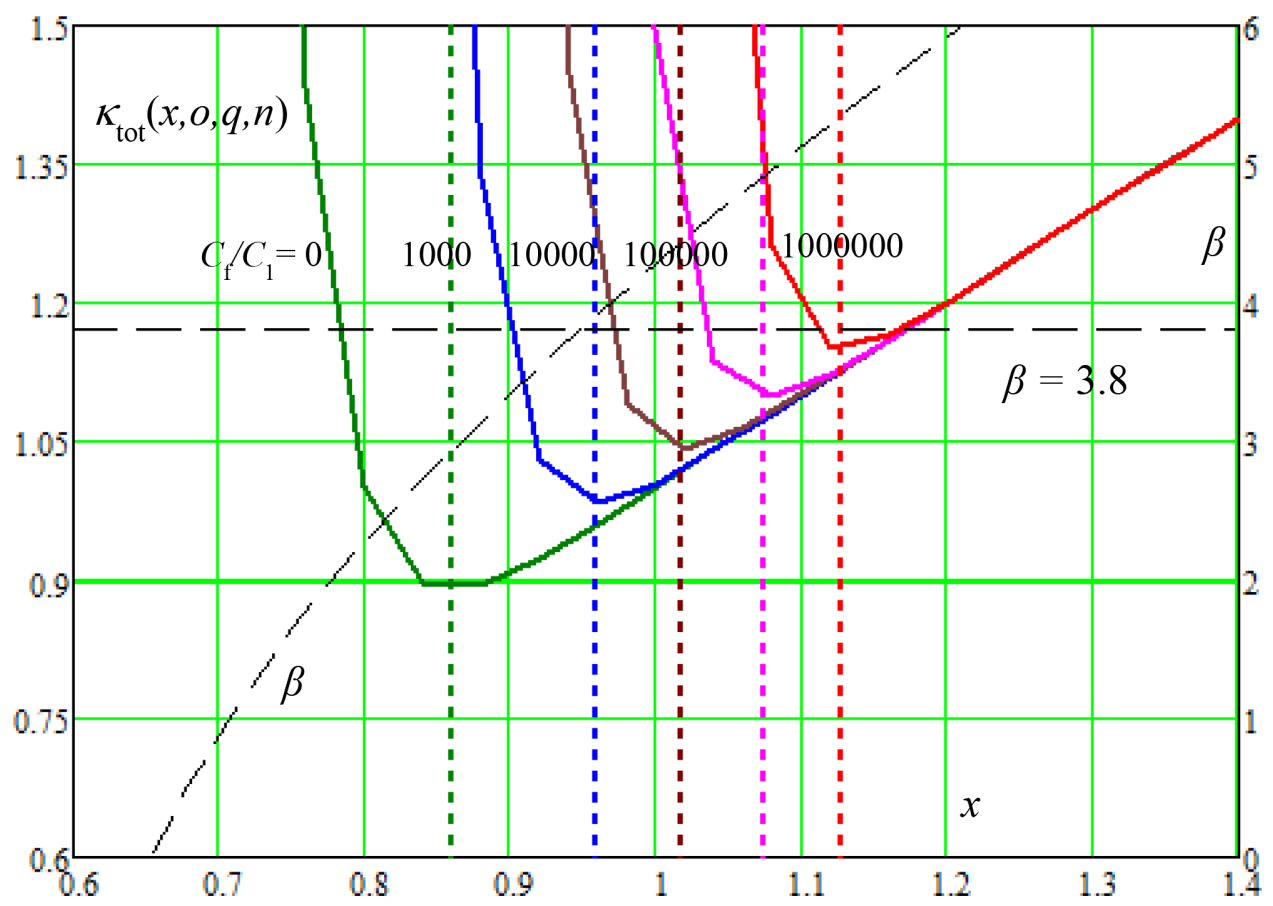

Figure 2: Variation of the total standardized cost $k_{\mathrm{tot}}(x, q, n)$ and the optimum reliability index

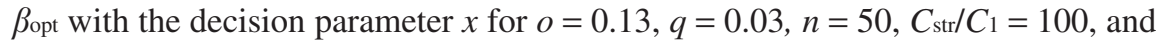
selected $C_{\mathrm{f}} / C_{1}=0,1000,10000,100000$ and 1000000 .

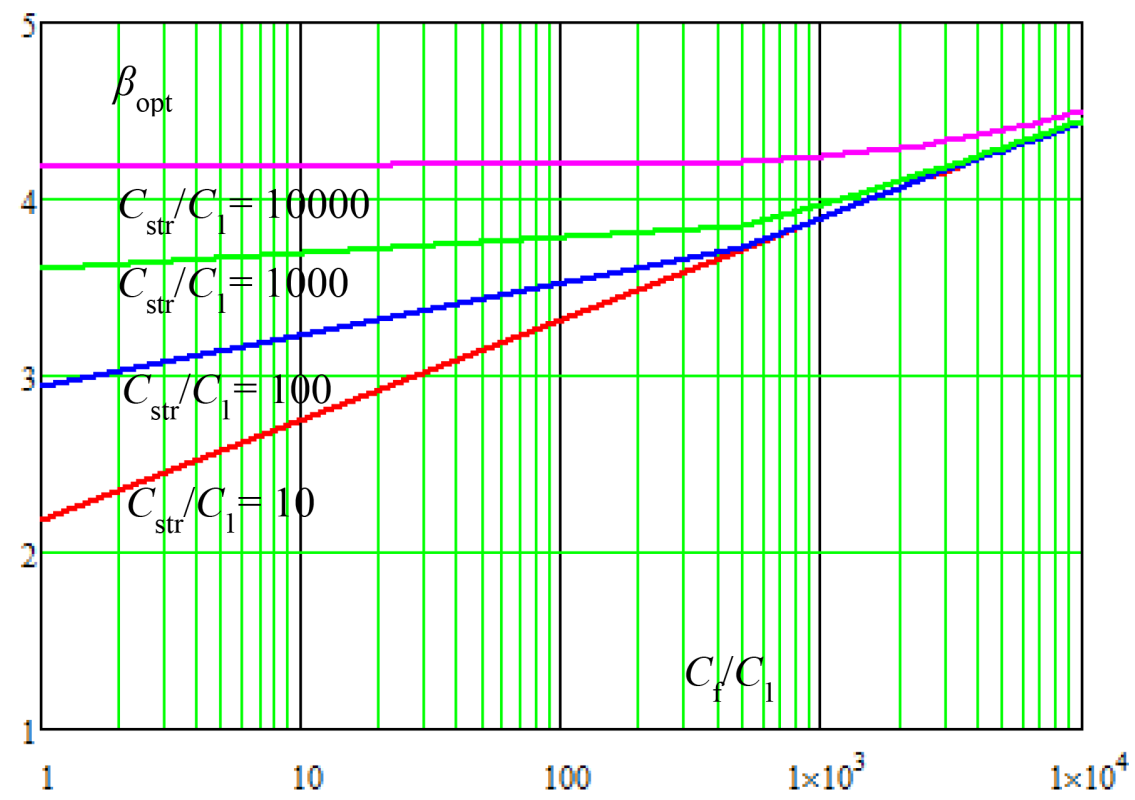

Figure 3: Variation of the optimum reliability index $\beta_{\text {opt }}$ with the cost ratio $C_{\mathrm{f}} / C_{1}$ for $o=0.13$, $q=0.03, n=50$ and selected $C_{\text {str }} / C_{1}=10,100,1000,10000$. 
In the example illustrated in Fig. 2, it is assumed that the rates $o=0.13$ is $q=0.03$, and the year number when the failure occurs is $n=50$. Under these assumptions, Fig. 2 shows the variation of the total standardized costs $\kappa_{\text {tot }}(x, o, q, n)$ (given by eqn (12)), and the optimum reliability index $\beta_{\text {opt }}$, with structural parameter $x$.

Figure 3 indicates variation of the optimum reliability index $\beta_{\text {opt }}$ with the cost ratio $C_{\mathrm{f}} / C_{1}$ for the rates $o=0.13, q=0.03$, number of years $n=50$ and selected $C_{\text {str }} / C_{1}=10,100,1000$, 10000 .

\section{CONCLUSIONS AND RECOMMENDATIONS}

The target reliability levels recommended in various national and international documents are inconsistent in terms of the values and the criteria according to which the appropriate values are to be selected. It is shown that the target reliability of structures can be derived from theoretical principles of probabilistic optimization considering the objective function as the total costs expressed as a sum of the initial costs $C_{0}$, the marginal costs $x C_{1}$ (where $x$ denotes the decision parameter and $C_{1}$ the incremental cost of decision parameter $x$ ), and the failure consequences consisting of the construction costs $C_{\text {str }}$ and failure costs $C_{\mathrm{f}}$ (the loss of structural utility at the time of failure $)$, these being taken into account by the cost ratios $\left(C_{\mathrm{str}} /\right.$ $\mathrm{C}_{1}$ and $C_{\mathrm{f}} / C_{1)}$.

The construction costs $C_{\text {str }}$ is discounted considering an annual obsolescence (oldness) rate $q$ and the time to failure (number of years) $n$, and the failure costs $C_{\mathrm{f}}$ is discounted considering an annual discount rate $q$ and the time to failure (number of years) $n$. In such a way the total cost is affected (reduced) by the obsolescence rate $o$ and discount rate $q$, and the number of years $n$.

An example of the probabilistic optimization of a generic structural member clearly shows (Figs 1 and 2) that the optimal reliability level, i.e. the reliability index $\beta$, depends primarily on:

- The construction costs $C_{\text {str? }}$,

- Failure costs (malfunctioning costs) $C_{\mathrm{f}}$,

- Costs for improving structural safety $C_{1}$.

The obsolescence (oldness) rate $o$ and discount rate $q$ and the time to failure $n$ seem to be less significant.

\section{ACKNOWLEDGEMENT}

This contribution has been developed as a part of the research project GAČR 16-11378S 'Risk based decision making in construction' supported by the Czech Grant Agency.

\section{REFERENCES}

[1] EN 1990 (2002) Eurocode/Basis of structural design, CEN.

[2] Diamantidis, D., "Reliability differentiation", In.: Holický et al.: Guidebook 1, Load effects on Buildings, CTU in Prague, Klokner Institute, ISBN 978-80-01-04468-1, pp. 48-61, 2009.

[3] ISO 2394., General principles on reliability for structures, International Organization for Standardization, Geneva, Switzerland, p. 73, 1998.

[4] JCSS., Joint Committee for Structural Safety. "Probabilistic Model Code", 2001, available at http://www.jcss.ethz.ch/ 
[5] Rackwitz, R., Optimization - the basis of code-making and reliability verification. Structural Safety, 22(1), pp. 27-60, 2000. https://doi.org/10.1016/S0167-4730(99)00037-5

[6] Fischer, K., Barnardo-Viljoen, C. \& Faber, M.H., Deriving target reliabilities from the LQI, LQI Symposium in Kgs. Lyngby, Denmark.

[7] Holický, M. \& Retief, J., Theoretical Basis of the Target Reliability. In 9th International Probabilistic Workshop. Braunschweig: Technische Universität, pp. 91-101, 2011, ISBN 978-3-89288-201-5.

[8] Holický, M., Reliability analysis for structural design, SUN MeDIA Stellenbosch, ZA, ISBN 978-1-920338-11-4, p. 199.

[9] Holický, M. \& Schneider, J., Structural design and reliability benchmark study. In Safety, Risk and Reliability - Trends in Engineering clo IABSE, ETH Zürich, International Conference in Malta, ISBN 3-85748-102-4, pp. 929-938, 2009. 